\title{
Modeling of the interaction of the layered barrier with a high-speed fragment
}

\author{
Alexandr Ishchenko ${ }^{1}$, Nikolay Belov ${ }^{1}$, Viktor Burkin ${ }^{1}$, Anton Sammel ${ }^{1, *}$, Nikolay Yugov $^{1}$, \\ and Marat Khabibullin ${ }^{1}$ \\ ${ }^{1}$ National Research Tomsk State University, 36, Lenin Ave., Tomsk, 634050 Russia
}

\begin{abstract}
For the manufacture of transparent armor of high class protection, as a rule, reinforced silicate glasses, as well as transparent ceramics, are used. Since these materials are resiliently brittle, they can be used only in transparent multilayered barriers with protective back films for protection against high-speed fragmentation elements and bullets. Plexiglass or polycarbonate is most often used as the back layer. The barrier's face layer must have a hardness substantially higher than the hardness of the drummer's material, and the Hugonievskii elasticity limit must exceed the shock-wave pressure arising at the initial stage in the barrier. The purpose of this paper is to develop a mathematical model that allows, within the framework of a porous elastic-plastic medium with regard to various fracture mechanisms, to calculate the impact interaction of fragmentation elements with transparent armor. Numerical research was conducted with the help of copyright software systems. Experimental studies of the collision of transparent armor with a splinter simulator in the speed range of $1500 \ldots 2500 \mathrm{~m} / \mathrm{s}$ were carried out with the use of throwing installations of the NRI AMM TSU.
\end{abstract}

\section{Introduction}

For the manufacture of transparent armor of high class protection, as a rule, multilayered structures with protective backing films that retain the fragments are used. Structural glass ceramics based on silicate glass, silica and alumina, etc., are mainly used as materials for the transparent armor manufacture. A promising option for creating transparent armor can be the use of a plate of a leucosapphire monocrystal as a face layer, but its cost is very high and there are limitations in the possibility of obtaining large leucosapphires. The barrier's face layer must have a hardness substantially higher than the hardness of the drummer's material, and the Hugonievskii elasticity limit must exceed the shock-wave pressure arising at the initial stage in the barrier. Only in this case is it possible to ensure the hardness of the barrier, sufficient to destroy the drummers without penetrating the barrier. In this regard, there is development of transparent polycrystalline materials. One of the leading materials of this kind is aluminum oxynitride (ALON), in its strength it is similar to leucosapphire. Plexiglass (PMMA) or polycarbonate, which combine high strength and rigidity with high impact resistance, is most often used as the back layer.

\footnotetext{
${ }^{*}$ Corresponding author: anton_sammel@mail.ru
} 
Designing optimal compositions to increase the transparent armor's resistance is impossible without prior mathematical modeling of the interaction processes with a highspeed drummer, imitating fragments or bullets.

The purpose of this paper is to develop a mathematical model that allows, within the framework of a porous elastic-plastic medium [1] with regard to various fracture mechanisms, to calculate the impact interaction of metal fragments with the transparent armor's elements, a layer of plexiglass in particular. A series of experiments necessary for verification of the mathematical model in a wide range of speeds was carried out on an experimental ballistic complex [2] equipped with a $23 \mathrm{~mm}$ caliber smooth-bore ballistic installation.

\section{The results of experimental and theoretical studies}

To verify the model of behavior of structural materials under high-speed impact, a series of experiments was conducted to study the interaction of a steel spherical drummer with a diameter of $5.85 \mathrm{~mm}$ (Fig. 1 a) with an $8 \mathrm{~mm}$ thick PMMA layer (Fig. $1 \mathrm{~b}$ ) in the speed range of $1500 \ldots 2500 \mathrm{~m} / \mathrm{s}$.

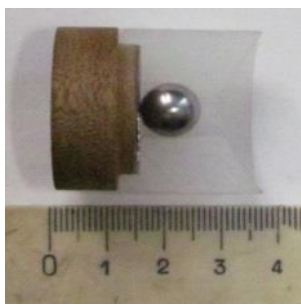

a)

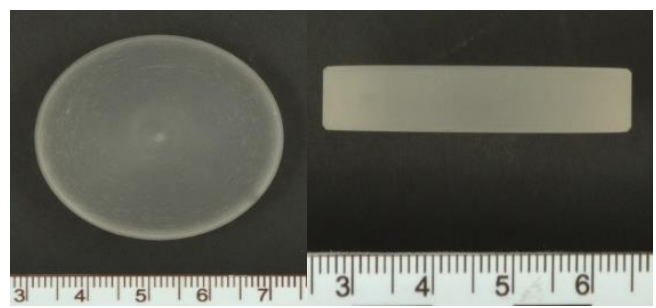

b)

Fig 1. A throwing setup with spherical drummer (a) and a sample of PMMA (b)

Fig. 2. demonstrates a photograph of a $23 \mathrm{~mm}$ caliber ballistic installation

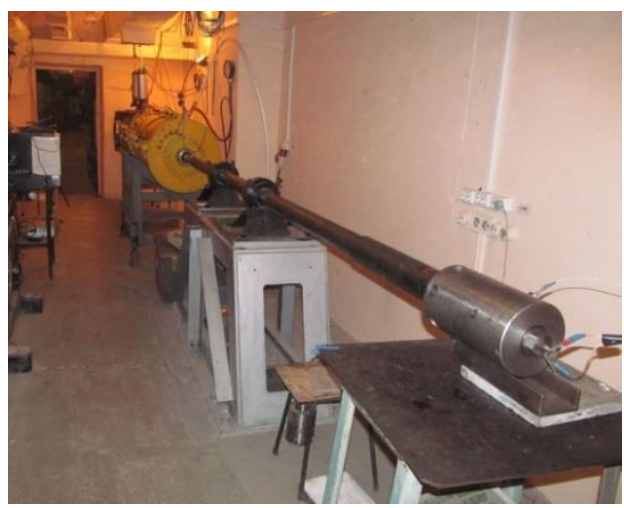

Fig 2. Ballistic installation

To determine the sample's armor resistance, a composite barrier was used: the first layer of the PMMA test material, the second one, the barrier-"witness" made of D16 aluminum alloy, at a distance of $60 \mathrm{~mm}$ (Fig. 3). The spherical drummer, after penetrating the test sample, interacted with the barrier-"witness" and left the crater. According to the results of high-speed video filming, the drummer's movement speed is measured before and after interaction with the test material sample. 


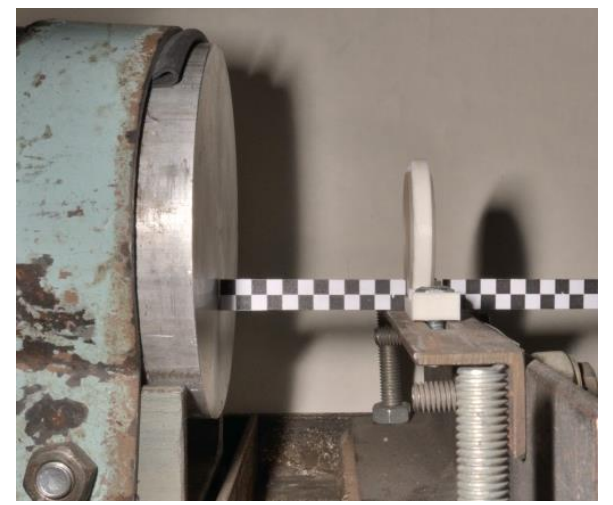

Fig 3. The locations of plexiglass and the barrier-"witness"

Fig. 4a shows the interaction of a spherical drummer with plexiglass at a speed of $V_{0}=$ $1595 \mathrm{~m} / \mathrm{s}$.
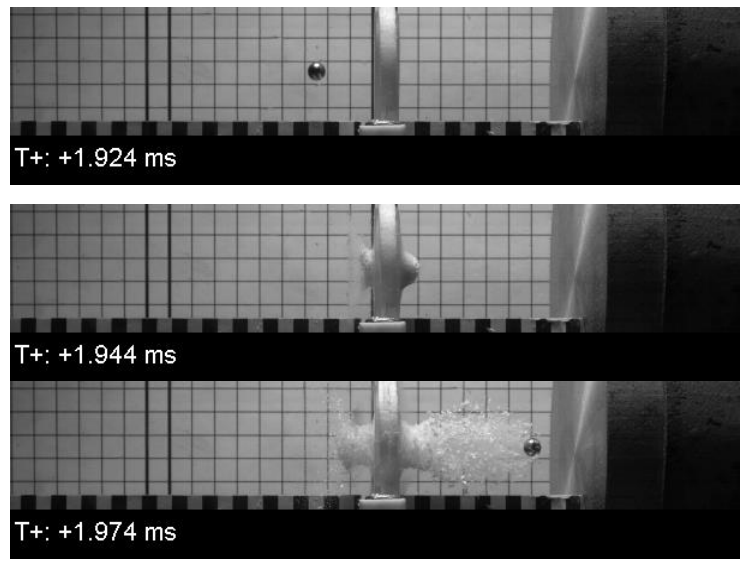

a)
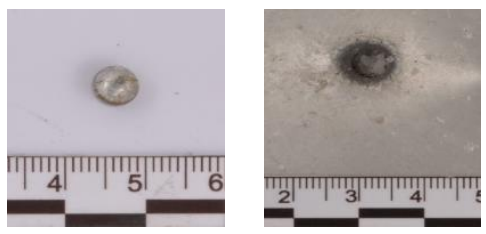

b)

c)

Fig 4. The photographic record of the plexiglass penetration $(a)$, the drummer $(b)$ and the front layer of the barrier-"witness" $(c)$ after the collision at $V_{0}=1595 \mathrm{~m} / \mathrm{s}$

The interaction of a spherical element with plexiglass and the ejection of material on the back side of the sample occurs at an interaction time $t=1.94 \mathrm{~ms}$. As can be seen from the figure at a time $t=1.97 \mathrm{~ms}$, the spherical drummer breaks through the sample under test. The drummer's speed after breaking through the plexiglass is $V=1330 \mathrm{~m} / \mathrm{s}$. In this experiment, at the moment of interaction with the barrier - "witness", the drummer is deformed to the state of a flat disk (Fig. 4 b). There forms a crater with a depth of $h=19.44$ $\mathrm{mm}$ in the barrier-"witness".

Fig. 5 shows the results of mathematical modeling of the interaction of a steel spherical drummer with transparent armor under conditions that repeat the experiment presented above. The calculations were carried out in axisymmetric formulation, with the use of a software package [3]. The spherical pores growth model is used as a fracture model [4]. The criterion of destruction is the achievement of porosity of the critical value $\alpha *$. Table 1 shows the parameters of the mathematical model of the materials under study, where denoted: $\rho_{0}$ is the initial material density, $\rho_{\mathrm{m} 0}, c_{\mathrm{m} 0}, S_{\mathrm{m} 0}, \gamma_{\mathrm{m} 0}, \mu_{\mathrm{m} 0}, \sigma_{\mathrm{ms}}$ are the parameters of the materials matrix, $a_{\mathrm{s}}, \alpha_{00}, \alpha_{0}, \alpha^{*}$ are the parameters of the destruction model. 
Table 1. The mathematical model parameters.

\begin{tabular}{|c|l|l|c|c|c|c|c|c|c|l|}
\hline Material & $\begin{array}{c}\rho_{m 0}, \\
\mathrm{~g} / \mathrm{cm}^{3}\end{array}$ & $\begin{array}{c}c_{m 0}, \\
\mathrm{~cm} / \mathrm{mcs}\end{array}$ & $S_{m 0}$ & $\gamma_{m 0}$ & $\begin{array}{c}\mu_{m 0}, \\
\mathrm{Mbar}\end{array}$ & $\begin{array}{c}\sigma_{s}, \\
\mathrm{Mbar}\end{array}$ & $\begin{array}{c}a_{s}, \\
\mathrm{Mbar}\end{array}$ & \multicolumn{1}{|c|}{$\alpha_{00}$} & \multicolumn{1}{|c|}{$\alpha_{0}$} & $\alpha^{*}$ \\
\hline Steel & 7.85 & 0.457 & 1.49 & 2.0 & 0.79 & - & 0.0043 & 1.0006 & 1.0006 & 1.43 \\
\hline PMMA & 1.18 & 0.257 & 1.54 & 1.9 & 0.0119 & 0.00026 & 0.00011 & 1.00001 & 1.00001 & 1.1 \\
\hline D16 & 2.78 & 0.5386 & 1.284 & 2.1 & 0.277 & - & 0.0027 & 1.0002 & 1.0002 & 1.43 \\
\hline
\end{tabular}

The results are presented in the form of a velocity field with respect to the maximum velocity $u_{\max }$ (left) and pressure field (right) in a cylindrical coordinate system $x, z,(\mathrm{~cm})$ at various points in time $t$ of the interaction between the drummer and the barrier. It also shows the values of the maximum velocity $u_{\max }$ and the velocity of the drummer's center of mass $u_{\mathrm{cm}}$.

At the time moment $t=12.0 \mathrm{mcs}$ the drummer pierces the sample, without deforming, and forms the ejection of the material. When interacting with the barrier at the time moment $t=50.0 \mathrm{mcs}$ the drummer deforms, taking the form of a disk, and gets stuck in it. The depth of the crater formed is $h=20.07 \mathrm{~mm}$. The accordance between the experiment and the calculation on velocity after interaction with sample is $4.1 \%$, on the crater depth is $3.2 \%$.

$t=0.9 \mathrm{mcs}$, $u_{\max }=1544 \mathrm{~m} / \mathrm{s}$, $u_{\mathrm{cm}}=1520 \mathrm{~m} / \mathrm{s}$

$t=3.9 \mathrm{mcs}$, $u_{\max }=1461 \mathrm{~m} / \mathrm{s}$, $u_{\mathrm{cm}}=1429 \mathrm{~m} / \mathrm{s}$

$t=12$ mcs, $u_{\max }=1387 \mathrm{~m} / \mathrm{s}$, $u_{\mathrm{cm}}=1385 \mathrm{~m} / \mathrm{s}$

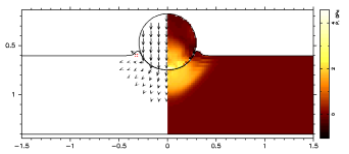

$t=13 \mathrm{mcs}$, $u_{\max }=1383 \mathrm{~m} / \mathrm{s}$, $u_{\mathrm{cm}}=1308 \mathrm{~m} / \mathrm{s}$

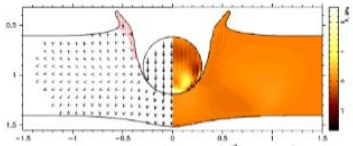

$t=32 \mathrm{mcs}$, $u_{\max }=480 \mathrm{~m} / \mathrm{s}$, $u_{\mathrm{cm}}=474 \mathrm{~m} / \mathrm{s}$

$t=50 \mathrm{mcs}$, $u_{\max }=13 \mathrm{~m} / \mathrm{s}$, $u_{\mathrm{cm}}=2 \mathrm{~m} / \mathrm{s}$

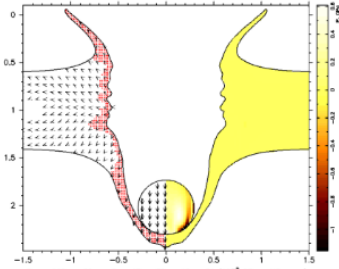

a)
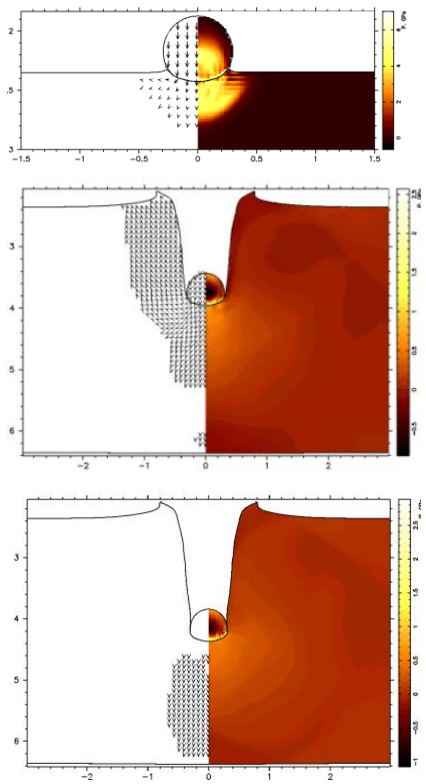

b)

Fig 5. The chronogram of the interaction process between the drummer and the barrier made from PMMA (a), and with the barrier-"witness" $(b)$ at $V_{0}=1595 \mathrm{~m} / \mathrm{s}$

The following experiment was carried out at an interaction speed $V_{0}=2014 \mathrm{~m} / \mathrm{s}$. Fig. 6 shows the results of the interaction of a steel spherical drummer with a plexiglass sample.

At the time moment $t=1.55 \mathrm{~ms}$, the drummer breaks through the sample and forms an outburst of material on the back side of the barrier. The drummer's speed after breaking through the plexiglass is $V=1722 \mathrm{~m} / \mathrm{s}$. Having passed the sample completely, there is no change in the state of the spherical drummer when approaching the barrier-"witness". After interacting with the barrier, the drummer crumbled into large fragments (Fig. 6 b), forming a crater with a depth of $h=22.22 \mathrm{~mm}$ (Fig. $6 \mathrm{c}$ ). 

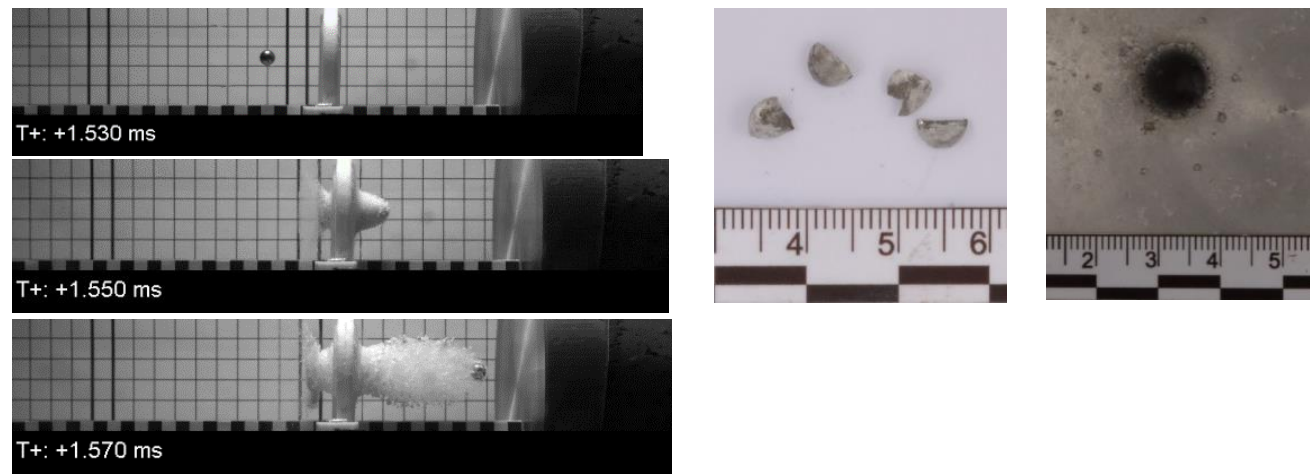

a)

b)

c)

Fig 6. The photographic record of the plexiglass penetration $(a)$, the drummer fragments $(b)$ and the front of the barrier-"witness" (c) after the collision at $V_{0}=2014 \mathrm{~m} / \mathrm{s}$

The simulation of this experiment is shown in Fig. 7. In this calculation, at time $t=10.0$ mcs, as in the experiment, material is ejected from the back of the sample. The destruction of the drummer into fragments under the conditions of modeling in an axisymmetric formulation cannot be described. However, the figure shows the zone of tensile strains, which indicates the material destruction. The depth of the crater formed is $h=21.28$. The accordance with the experiment on the depth is $4.2 \%$, on the velocity after the interaction with sample - complete match.

$t=0.9 \mathrm{mcs}$,
$u_{\max }=1916 \mathrm{~m} / \mathrm{s}$,
$u_{\mathrm{cm}}=1882 \mathrm{~m} / \mathrm{s}$

$t=3.9 \mathrm{mcs}$,

$u_{\max }=1796 \mathrm{~m} / \mathrm{s}$, $u_{\mathrm{cm}}=1754 \mathrm{~m} / \mathrm{s}$

$t=10 \mathrm{mcs}$, $u_{\max }=1728 \mathrm{~m} / \mathrm{s}$, $u_{\mathrm{cm}}=1722 \mathrm{~m} / \mathrm{s}$

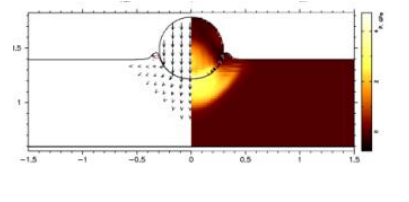

$$
\begin{aligned}
& t=11 \mathrm{mcs}, \\
& u_{\max }=427 \mathrm{~m} / \mathrm{s}, \\
& u_{\mathrm{cm}}=418 \mathrm{~m} / \mathrm{s}
\end{aligned}
$$

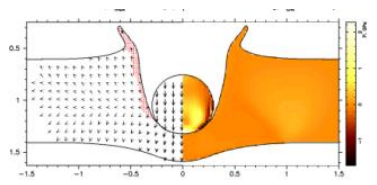

$t=32 \mathrm{mcs}$, $u_{\text {max }}=427 \mathrm{~m} / \mathrm{s}$, $u_{\mathrm{cm}}=418 \mathrm{~m} / \mathrm{s}$

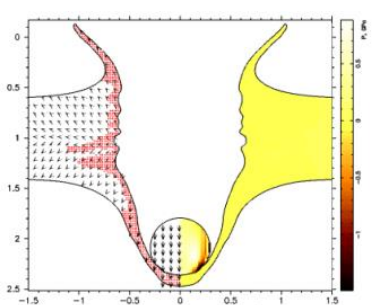

a)
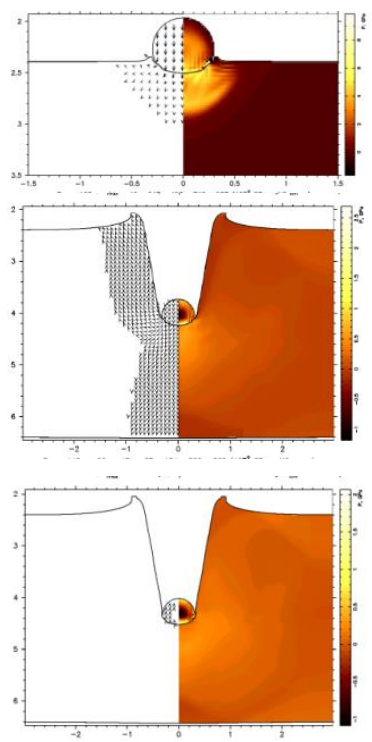

b)

Fig 7. The chronogram of the interaction process between the drummer and the barrier made from PMMA (a), and with the barrier-"witness" $(b)$ at $V_{0}=2014 \mathrm{~m} / \mathrm{s}$

The last experiment presented in fig. 8 shows the interaction of a spherical drummer with plexiglass at speed $V_{0}=2441 \mathrm{~m} / \mathrm{s}$. 

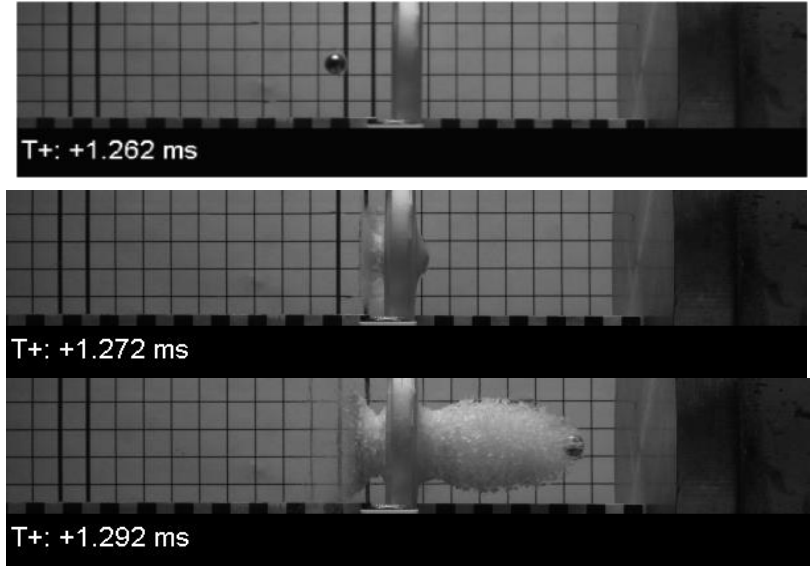

$a$

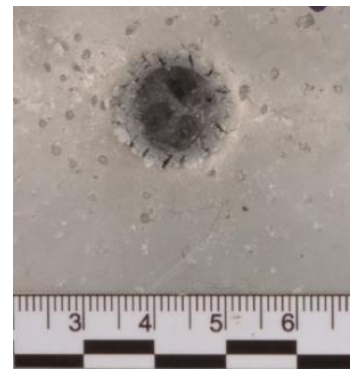

$b$

Fig 8. The photographic record of the plexiglass penetration $(a)$, and the front of the barrier-"witness" (b) at $V_{0}=2441 \mathrm{~m} / \mathrm{s}$

The spherical drummer, when breaking through the sample, forms a small convexity on the back side at the time moment $t=1.27 \mathrm{~ms}$. In this experiment there is no significant change in the shape of the drummer after breaking through the plexiglass. The drummer's speed after breaking through the plexiglass is $V=2048 \mathrm{~m} / \mathrm{s}$. After interacting with the barrier, the drummer crumbles into small fragments and leaves a crater with a depth of $h=$ $15.03 \mathrm{~mm}$. The decrease in the crater depth in comparison with the previous experiments is due to the fact that most of the energy necessary for the formation of the crater was used for the destruction of the drummer.

$t=0.9 \mathrm{mcs}$,
$u_{\max }=2337 \mathrm{~m} / \mathrm{s}$,
$u_{\mathrm{cm}}=2282 \mathrm{~m} / \mathrm{s}$

$t=3.9 \mathrm{mcs}$,

$u_{\text {max }}=2131 \mathrm{~m} / \mathrm{s}$, $u_{\mathrm{cm}}=2097 \mathrm{~m} / \mathrm{s}$

$t=7.9 \mathrm{mcs}$, $u_{\text {max }}=2089 \mathrm{~m} / \mathrm{s}$, $u_{\mathrm{cm}}=2068 \mathrm{~m} / \mathrm{s}$

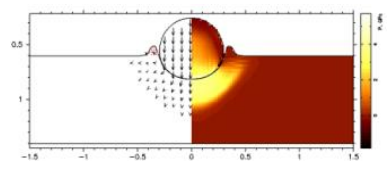

$$
\begin{aligned}
& t=9 \mathrm{mcs}, \\
& u_{\max }=2026 \mathrm{~m} / \mathrm{s}, \\
& u_{\mathrm{cm}}=1779 \mathrm{~m} / \mathrm{s}
\end{aligned}
$$

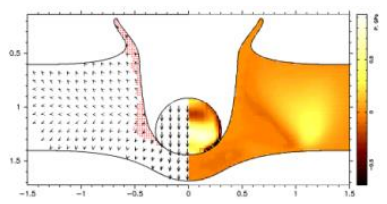

$$
t=22 \mathrm{mcs} \text {, }
$$
$u_{\max }=490 \mathrm{~m} / \mathrm{s}$, $u_{\mathrm{cm}}=470 \mathrm{~m} / \mathrm{s}$

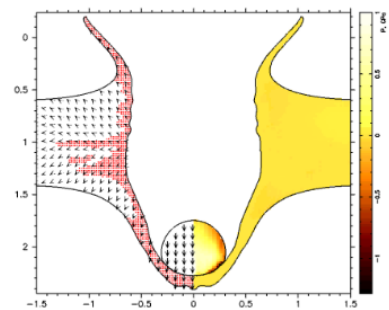

a)
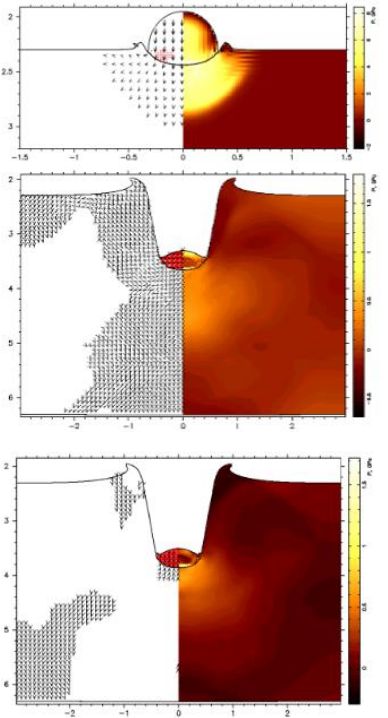

b)

Fig 9. The chronogram of the interaction process between the drummer and the barrier made from PMMA $(a)$, and with the barrier-"witness" $(b)$ at $V_{0}=2441 \mathrm{~m} / \mathrm{s}$

Fig. 9 shows the results of mathematical modeling of the interaction of the drummer with the barrier, repeating the experimental conditions presented above. It can be seen from 
the calculations that at time $t=7.9$ mcs the spherical drummer, as in the experiment, forms a convexity on the back side in the test sample. The spherical element itself, when breaking through the barrier, is deforming, starting to take the form of a disk. When interacting with the barrier, the spherical drummer is destroyed by a shear mechanism. The depth of the crater formed is $h=15.51 \mathrm{~mm}$. The accordance between the calculation and the experiment on velocity after the interaction with a sample is $1.0 \%$, on the crater depth is $3.2 \%$.

Thus, the presented mathematical model of high-speed collision of transparent armor with a steel fragment proved to be useful. As can be seen from the experiments, the spherical element at the interaction speed from $2000 \mathrm{~m} / \mathrm{s}$ begins to crumble into fragments. In the calculations, zones of the formation of tensile strains are just visible. Therefore, at an interaction speed of $V_{0}=2441$, the crater depth was less than in the experiment at a speed of $V_{0}=2014 \mathrm{~m} / \mathrm{s}$, as all the energy needed to form a crater was used for the destruction of the spherical drummer.

\section{References}

1. N.N. Belov, V.N. Demidov, L.V. Efremov et al. Izvestia vuzov. Physics, 35, 8, 5- 49 (1992) (in Russian)

2. V.A. Burakov, V.V. Burkin, A.N. Ishchenko et al. Patent for invention (No. 2591132 dated June 20, 2016)

3. M.V. Khabibullin, S.A. Afanasyeva. The calculation of phenomena occurring in condensed matter as a result of intense impulse effects in axisymmetric formulation. Federal Service for Intellectual Property. Certificate of state registration of computer programs. (No. 2012617301. M., 2012) (in Russian)

4. N.N. Belov, A.I. Korneev, A.P. Nikolaev. Numerical analysis of plates failure under the influence of impulse loads, PMTF, 3, 132-136 (1985) 